\title{
Visão Geral da Sísmica e seu uso na Estimativa de Reservas
}

Marcos Hexsel Grochau, Petrobras

Vitor Leal de Mello, Petrobras

Gina Claudia Nadal Nunes, Petrobras

Copyright 2014, SBGf - Sociedade Brasileira de Geofísica

Este texto foi preparado para a apresentação no VI Simpósio Brasileiro de Geofísica, Porto Alegre, 14 a 16 de outubro de 2014. Seu conteúdo foi revisado pelo Comitê Técnico do VI SimBGf, mas não necessariamente representa a opinião da SBGf ou de seus associados. É proibida a reprodução total ou parcial deste material para propósitos comerciais sem prévia autorização da SBGt.

\section{Resumo}

A utilização de dados sísmicos na indústria do petróleo tem crescido muito nas últimas décadas. A sísmica tem contribuído na descoberta, desenvolvimento e monitoramento dos reservatórios. Pode fornecer informações valiosas tais como o volume rochoso, compartimentalizações por falhas, contato entre fluidos, pressões entre blocos e outras. Este trabalho visa apresentar uma visão geral das técnicas sísmicas assim como associá-las a um dos mais importantes indicadores de uma companhia de petróleo: as reservas de hidrocarbonetos. Procurou-se simplificar os termos para transcender disciplinas assim como apresentar a relação sísmica - reservas em dois eixos: (a) redução de incertezas e (b) maturidade do projeto.

\section{Introdução}

$\mathrm{Na}$ indústria do petróleo, os principais indicadores de desempenho de uma empresa são produção e reservas. As reservas, volume de hidrocarbonetos que pode ser técnica e economicamente produzido, são, ao contrário da produção, difíceis de estimar. Os critérios, tais como da SEC (Securities and Exchange Commission, 2009) e PRMS (Petroleum Resources Management System, 2007) de reservas permitem a utilização de "tecnologias confiáveis"1 para apropriação de reservas. Os métodos geofísicos, e em especial a Sísmica, são alguns dos instrumentos que podem ser empregados. A utilização da Sísmica na estimativa de reservas vem, portanto, aumentando ao longo do tempo de acordo com sua evolução tecnológica e a própria evolução dos critérios de reservas.

Os dados sísmicos eram usados inicialmente apenas para comprovar a delimitação / extensão do reservatório e o tipo de trapa (estrutural ou estratigráfica). Com a evolução das técnicas relacionadas ao método sísmico, a contribuição destes dados na estimativa de reservas aumentou, podendo ser expresso e melhor compreendido

\footnotetext{
${ }^{1}$ Tradução literal do termo Reliable Technology adotado pela SEC na modernização de seus critérios, em 2009, para se referir a um grupo de uma ou mais tecnologias (incluindo métodos computacionais) que foram testadas em campo e demonstraram fornecer resultados razoavelmente certos com consistência e repetitividade, na fornecer resultados razoavelmente certos comán avaliada ou em reservatório análogo.
}

separando sua atuação em 2 eixos: o das incertezas técnicas e o da maturidade dos projetos (Figura 1) (Kloosterman, 2012).

No eixo horizontal, a sísmica contribui para avaliar e mitigar a incerteza técnica dos projetos de exploração e produção. O aumento na densidade de informações e a crescente melhoria da qualidade sísmica, além da associação com dados de geologia e engenharia, permitem reduzir a incerteza tanto dos volumes originais quanto dos volumes recuperáveis, permitindo, por exemplo, a recategorização de volumes de reservas de possível para provável e, finalmente, para provada.

Sabe-se, contudo, que os dados sísmicos possuem um grau de incerteza que é intrínseca ao método e relacionada à dificuldade de reconstituir a correta propagação da onda. Existem as incertezas na conversão tempo - profundidade, na anisotropia de velocidades, viés amostral, incerteza conceitual e de interpretação, entre outras.

No eixo vertical, é representado o grau de maturidade do projeto. Para a classificação do mesmo como Recurso Prospectivo, Recurso Contingente e Reserva, entre outros fatores, pode-se utilizar as informações obtidas através de tecnologias sísmicas. O nível de robustez das informações que a sísmica consegue acessar, tais como volume de rocha, saturação de fluidos e conectividade entre blocos contribuem para a classificação dos projetos nos diversos níveis de maturidade.
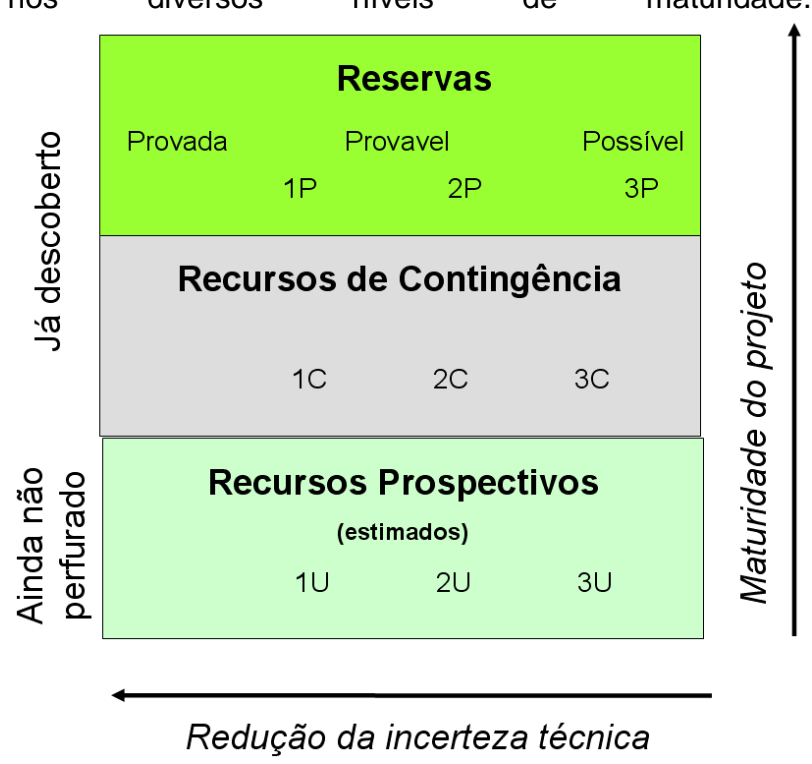

Figura 1 - Classificação de Reservas em relação à incerteza técnica e à maturidade do projeto. 
O objetivo deste trabalho é mostrar, de maneira didática e simplificada, os diversos aspectos do método sísmico que têm sido usados e que vem contribuindo para a estimativa das reservas de óleo e gás.

\section{Sísmica para Reduzir Incertezas nos Projetos}

O uso da sísmica na classificação e quantificação das reservas e recursos dá-se ao longo de todo o período de desenvolvimento da acumulação. Os dados sísmicos, depois de calibrados com as informações "pontuais" dos perfis de poços, amostras de rocha e dados de engenharia, permitem extrapolar esses dados no espaço.

A evolução da qualidade dos dados sísmicos está relacionada principalmente às novas técnicas de aquisição, tais como OBC ou Nodes (Figura 2), ao aumento da densidade de informação, à amostragem multiazimutal, e aos novos algoritmos de processamento.

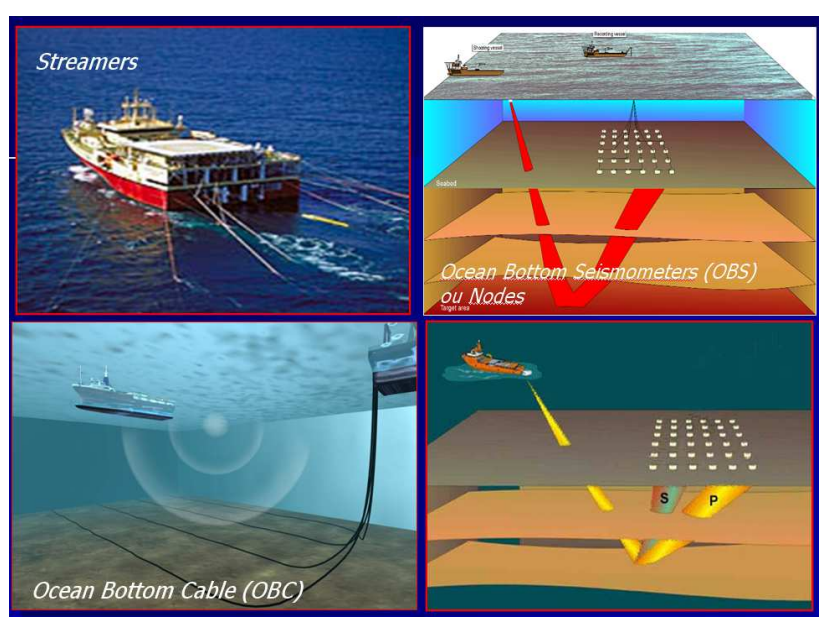

Figura 2 - Diferentes técnicas de aquisição sísmica que contribuem para a melhoria da qualidade do dado.

Desta forma, estando disponíveis volumes de dados sísmicos de boa qualidade, pode-se reduzir as incertezas na determinação tanto do volume rochoso como da presença e conectividade dos fluidos existentes no reservatório. A integração de dados de geologia e engenharia também contribui para a redução das incertezas em todas as etapas do desenvolvimento da jazida. Estes são insumos para uma série de atualizações nos modelos do reservatório que visam ser preditivos em relação aos volumes a serem produzidos (Kloosterman \& Pichon, 2012).

Esses modelos descrevem a geometria das trapas, delimitam a extensão do reservatório e indicam a presença de falhas. Apoiado nessas características iniciais o modelo é populado com uma distribuição de propriedades como a porosidade, a permeabilidade, a pressão e as saturações dos diferentes fluidos presentes nos poros.
Existem, porém, incertezas inerentes ao próprio método sísmico que devem ser explicitadas e consideradas. $O$ dado sísmico é adquirido ao longo do tempo, e sua conversão para profundidade requer um modelo de velocidade que não é perfeitamente conhecido. A anisotropia de velocidades muitas vezes não é capturada devido à geometria de aquisição de dados utilizada. No mar, por exemplo, predominam aquisições tipo streamers que são normalmente biazimutais (narrow azimuth). $\mathrm{O}$ sinal sísmico registrado relaciona-se às interfaces entre camadas, e faz-se necessário realizar o processo de inversão sísmica obtendo propriedades das camadas, tais como impedância, da qual se pode derivar a porosidade. O conteúdo de freqüência presente nos dados sísmicos não é suficientemente amplo para imagear camadas muito delgadas a grandes profundidades.

Apesar das limitações inerentes ao método, a evolução da qualidade do dado sísmico - associada às novas técnicas - tem permitido reduzir as incertezas na estimativa dos volumes de reservas e recursos prospectivos. Neste sentido, observa-se que a utilização da sísmica contribui para reduzir as incertezas contidas nos volumes recuperáveis e, consequentemente, a recategorização de possível para provável e desta para provada à medida que o projeto é desenvolvido.

Associando as incertezas sísmicas com outras do projeto, podem ser criados cenários de recuperação otimista, realista e pessimista para cada projeto. A estes cenários, após a declaração de comercialidade, marco regulatório no Brasil para apropriação de reservas, podem ser associadas reservas, assim denominadas: 3P (provada + provável + possível), 2P (provada + provável) e 1P (provada), respectivamente (PRMS, 2007).

\section{Sísmica relacionada à maturidade dos Projetos}

No eixo da maturidade dos projetos são definidas três classes: Reservas, Recursos Contingentes e Recursos Prospectivos. Essas classes estão relacionadas ao grau de comercialidade da acumulação. Reservas e Recursos Contingentes são classificações dadas sobre uma descoberta. De forma resumida, pode-se definir uma descoberta como uma comprovação por ao menos um poço de que existe uma acumulação significativa de hidrocarbonetos potencialmente móveis (Lorenzen et al., 2012).

Em uma área exploratória, porém sem poço descobridor, uma potencial acumulação é chamada de Recurso Prospectivo. Após a descoberta de uma acumulação, seu volume recuperável pode ser classificado como Recurso Contingente até que seja comprovada que sua explotação é técnica e economicamente viável, passando a ser classificada como Reserva.

Uma etapa inicial na utilização dos dados sísmicos que agrega informação na classificação/quantificação de 
recursos e reservas é a interpretação de horizontes e falhas. A interpretação do topo e da base delimita 0 volume rochoso de um dado reservatório. A definição dos contatos entre fluidos fornece uma estimativa do volume ocupado pelas rochas saturadas por cada tipo de fluido.

O mapeamento das falhas tem uma grande importância para identificar possíveis separações entre zonas produtoras e selos no reservatório. No caso onde o reservatório é dividido por uma falha potencialmente selante, ou quando seu rejeito é mais espesso que o reservatório, é necessário ao menos um poço em cada bloco para a classificação de suas acumulações como Reservas. Se apenas um bloco possui poço perfurado, a região além da falha é classificada como Recurso Prospectivo. Outro ponto a ser observado é a continuidade ou não do contato entre fluidos de um bloco para o outro.

Os atributos sísmicos, em vários casos, mostram-se como uma importante ferramenta na interpretação de falhas e da continuidade do reservatório. Voltando ao cenário do reservatório separado por uma falha de grande rejeito, se no bloco onde há poços perfurados as anomalias de amplitude respondem bem à presença de hidrocarbonetos, este atributo (amplitude) pode ser utilizados para estimar a continuidade da acumulação no outro bloco.

A análise de AVO (amplitude versus offset) é outra forma de correlacionar os dados sísmicos com propriedades do reservatório. Através de estudos sobre dados sísmicos pré-empilhamento, não é raro identificar correlações entre as amplitudes e o tipo de fluido. Com isso, essa ferramenta auxilia na interpretação do contato entre fluidos, da continuidade do reservatório e identificação de regiões desconectadas do reservatório principal (Lorenzen et al., 2012).

Além da amplitude sísmica, propriedade da interface, a impedância, propriedade de camada e derivada do processo de inversão sísmica, fornece informações valiosas da jazida (Figura 3). A partir de perfis de poços e ensaios laboratoriais em amostras de rocha, é possível que existam altas correlações entre impedância sísmica e propriedades do reservatório. Neste caso, a impedância pode guiar a distribuição dessa propriedade num modelo tridimensional. Um exemplo clássico de uso da inversão para geração de um modelo do reservatório é a correlação entre a impedância acústica no reservatório com os valores de porosidade. Outra grande contribuição da inversão sísmica se dá pelo fato de sua teoria prever a não unicidade de soluções para um mesmo problema. Desta forma, a variabilidade em seus resultados pode ser incorporada para dimensionar as incertezas nos modelos e, conseqüentemente, na definição de volumes declarados como Reservas e Recursos.

Além disso, os atributos de impedância, assim como os volumes em amplitude, são também utilizados para a interpretação das geometrias externa e interna do reservatório. Os dados invertidos normalmente apresentam maior resolução e menor razão sinal/ruído.

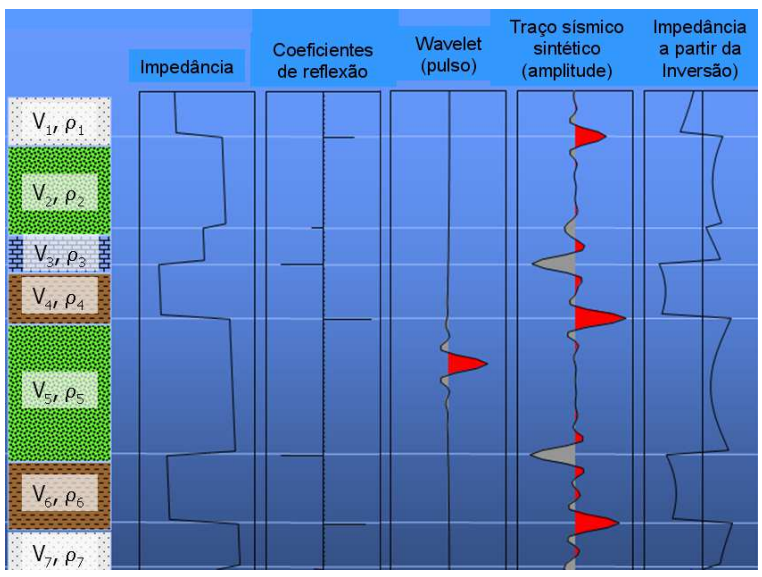

Figura 3 - Relação entre propriedades das camadas (impedância, produto da velocidade pela densidade) e das interfaces (amplitude sísmica).

Vale lembrar que os dados sísmicos são medidas de amplitudes de onda ao longo de um dado tempo de registro. Para a transformação da variável tempo para profundidade, e assim possibilitar a obtenção de uma estimativa da espessura do reservatório, é fundamental um modelo de velocidades. Uma variação no modelo de velocidades tem impacto direto no volume do reservatório e, conseqüentemente, no volume explotável.

\section{Outras Técnicas Aplicadas na Classificação de Reservas}

As técnicas aqui apresentadas são as mais comumente aplicadas na caracterização do reservatório. No entanto, ao longo da última década, outras aplicações da sísmica têm sido consideradas "tecnologias confiáveis". O monitoramento sísmico do reservatório durante toda a sua vida produtiva, por exemplo, vem ganhando cada vez mais força. Consiste na comparação entre volumes sísmicos adquiridos em diferentes estágios da produção de um campo (4D ou time-lapse) com o objetivo de identificar os efeitos da produção no interior ou entorno do reservatório.

Dados 4D, que englobam tanto variações de amplitude como de impedância (oriundos da inversão sísmica 4D), constituem importante ferramentas não apenas para a estratégia de gerenciamento do campo, mas também no âmbito da classificação/quantificação de Reservas e Recursos, desde que asseguradas a consistência e a repetitividade da técnica. Isso porque essas metodologias permitem delimitar áreas lavadas e não lavadas pelos poços injetores, mapear a variação no contato entre fluidos no reservatório, determinar se uma falha é selante ou não determinando se regiões do campo estão hidraulicamente conectadas (Figura 4), além de fornecer 
informações importantes para a atualização dos modelos geológicos e de fluxo do reservatório.

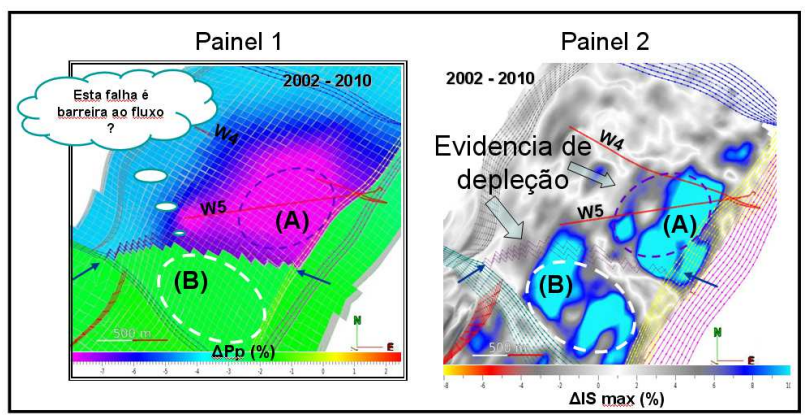

Figura 4 - Painel 1: modelo mostrando falha atuando como barreira ao fluxo entre as regiões $(A)$ e $(B)$; Painel 2: dados sísmico de impedância mostrando que as regiões estão comunicadas (anomalia azul) e que a falha não é barreira efetiva.

\section{Conclusões}

O processo de estimativa de reservas e recursos é de grande importância em toda companhia de petróleo. É papel do geofísico compreender como suas ferramentas de trabalho podem contribuir para o cumprimento desta tarefa. Há um amplo conjunto de técnicas aplicadas aos dados sísmicos que podem ser consideradas confiáveis, no âmbito de classificação, categorização e quantificação de reservas e recursos e de mitigação de incertezas dos projetos. A participação do geofísico nestas etapas tende a crescer com o desenvolvimento de novas tecnologias na área de sísmica.

Além do aprimoramento e desenvolvimento de novas técnicas voltadas para a caracterização sísmica dos reservatórios, há muito que ser explorado em relação às tecnologias de monitoramento da produção, como a sísmica 4D e os sistemas de monitoramento permanentes (PRM).

\section{Agradecimentos}

Gostaríamos de agradecer a PETROBRAS - Petróleo Brasileiro SA pela autorização para apresentação deste trabalho.

Agradecemos em particular ao geofísico Paulo Roberto Schroeder Johann e ao engenheiro Juan Antonio Molina de Araújo pelo incentivo em integrar as disciplinas de Sísmica e Reservas e apoiar esta publicação. Agradecemos também à engenheira Aline Barreto Oliveira pela valiosa revisão e sugestões.

\section{Referências}

Kloosterman, H.J., Pichon, PL, 2012. The role of geophysics in petroleum resources estimation and classification - new industry guidance and best practices. The Leading Edge, 2012 vol.31: 1034-1040.

Lorenzen, R., Kloosterman, H. J., Abriel, W., 2012. An introduction to this special section: Geophysics in reserves estimation. The Leading Edge, 2012 vol.31: 1024-1025.

Lorenzen, R., Purewal, S., Etherington, J., 2012. Introduction to the petroleum resources management system and the implications for the geophysical community. The Leading Edge, 2012 vol.31: 1028-1032.

Securities and Exchange Commission (SEC). Modernization of Oil and Gas Reporting; Final Rule, 2008. Disponível em: <http://www.sec.gov/rules/final/2008/338995.pdf>.

Society of Petroleum Engineers (SPE); World Petroleum Council (WPC); American Association of Petroleum Geologists (AAPG); Society of Petroleum Evaluation Engineers (SPEE). Petroleum Resources Management System (PRMS), 2007. Disponível em: <http://www.spe.org>. 